\title{
ANÁLISE ECONÔMICA DA PRODUÇÃO DE FRANGO DE CORTE SOB CONDIÇÕES DE RISCO NÖ ESTADO DO PARANÁ
}

\author{
Economical analysis of broilers production under risk conditions in the state of Paraná
}

\author{
Cármem Ozana de Melo ${ }^{1}$, Gerson Henrique da Silva ${ }^{1}$, Maura Seiko Tsutsui Esperancini²
}

\begin{abstract}
RESUMO
A avicultura de corte constitui-se numa importante atividade econômica no estado do Paraná e, como qualquer outra, está sujeita a riscos. Objetivou-se, neste trabalho, proceder à análise econômica da produção integrada de frango de corte, avaliando os riscos, considerando os sistemas climatizado, automático e manual. Utilizando-se as variáveis de risco: preço do produto, produtividade e custos de produção foi possível identificar as principais fontes de risco e sua influência na renda líquida. Os resultados apontaram que a rentabilidade da atividade é mais sensível aos componentes da receita do que de custos, sendo o preço a variável de maior sensibilidade. Verificou-se também que o aviário climatizado apresenta possibilidades de prejuízo mais alto para menores níveis de risco e, à medida que o risco aumenta, oferece retornos mais interessantes, em comparação aos sistemas automático e manual. $\mathrm{O}$ sistema manual foi o que passou a apresentar retorno a níveis de risco maior (acima de $25 \%$ ).
\end{abstract}

Termos para indexação: Análise de risco, avicultura, sistemas de produção, produção integrada, agroindústria.

\begin{abstract}
Poultry production is an important economic activity in the state of Paraná, and as any other, is under risk. In this context, economic analysis of broilers integrated management was employed to evaluate the risks, considering the acclimatized, automated and manual systems. By using the risk variables: price of product, productivity and costs of production, it was possible to identify the main risk sources and their influence on the net income. The results showed that the profitability of activity is more sensitive to the components of proceeds than to the costs, being the price the most sensitive variable. We also verified that the acclimatized poultry house has possibilities of higher loss for lower levels of risk, and the higher the risk the most interesting the earnings, in comparison to the automated and manual systems. The manual system was the one that presented earnings in higher levels of risk (above $25 \%$ ).
\end{abstract}

Index terms: Risk assessment, aviculture, management systems, integrated management, agroindustry.

(Recebido em 29 de janeiro de 2008 e aprovado em 06 de junho de 2008)

\section{INTRODUÇÃO}

A avicultura brasileira é bastante competitiva, configurando o país entre os três maiores produtores mundiais. Alia-se a isso o fato de que, desde seu surgimento e aperfeiçoamento para fins comerciais, a atividade tem promovido a criação de técnicas aprimoradas nos campos genético, operacional e de planejamento, com reflexos econômicos e sociais importantes, especialmente nos setores rural e agroindustrial.

Segundo Barros (2005), o processo de modernização e a produção em grande escala começaram na década de 1930 devido, principalmente, à necessidade de abastecer o mercado interno, já considerável na época. Na década de 1950 , ocorreram profundas modificações na avicultura brasileira, que ganhou novos impulsos com os avanços da genética, das vacinas, nutrição e equipamentos específicos para sua criação. O desenvolvimento dessa atividade a partir de 1950 deu-se, basicamente, nos estados do Sudeste. Na década de 1970 houve o deslocamento para a região Sul do país. As condições de produção de grãos, clima e de mãode-obra, ao lado do desenvolvimento da agroindústria do setor, colocaram o Brasil, em 2004, na posição de $3^{\circ}$ maior produtor mundial e líder nas exportações de carne de frango.

Desde 2000, o Paraná lidera a produção nacional de carne de frango, seguido dos estados de Santa Catarina, Rio Grande do Sul e São Paulo. Em 2003, participou com aproximadamente $22 \%$ do abate de frangos de corte com Serviço de Inspeção Federal (SIF). Em 2004, enquanto no Brasil houve crescimento no número de frangos abatidos da ordem de $8,85 \%$ em relação a 2003, no Paraná o aumento foi de $12,7 \%$, gerando um volume de carne de frango produzida da ordem de 1,831 milhão de toneladas. No que se refere ao mercado externo, em 2004, o Paraná ocupou o

${ }^{1}$ Economistas, Doutorandos em Energia na Agricultura - Faculdade de Ciências Agronômicas/FCA - Departamento de Gestão e Tecnologia Agroindustrial/ DGTA - Universidade Estadual Paulista/UNESP - Rua José Barbosa de Barros, 1780 - Fazenda Experimental Lageado - Cx. P. 237 - $18610-307$ Botucatu, SP - caromelo@fca.unesp.br; ghsilva@fca.unesp.br

2Engenheira Agrônoma, Doutora em Economia, Professora - Faculdade de Ciências Agronômicas/FCA - Departamento de Gestão e Tecnologia Agroindustrial/DGTA - Universidade Estadual Paulista/UNESP - Rua José Barbosa de Barros, 1780 - Fazenda Experimental Lageado - Cx. P. 237 18610-307 - Botucatu, SP - maura@fca.unesp.br 
$2^{\circ}$ lugar no ranking nacional, participando com $28,2 \%$ das exportações brasileiras, ultrapassando o estado do Rio Grande do Sul, com 25,3\% e vindo depois de Santa Catarina, com 44,8\% (SEAB/DERAL, 2005).

Em 2005, o frango de corte representou o segundo produto em arrecadação no valor bruto da produção agropecuária (VBP) estadual, perdendo apenas para a soja. São 72 municípios do estado que obtêm no frango a maior arrecadação do VBP, o que ratifica sua importância econômica e social, como fonte de divisas externas, geração de renda, empregos diretos e indiretos e fixação do homem no campo e interior do Estado (SEAB/DERAL, 2007a).

Já em 2007, segundo o Instituto Brasileiro de Geografia e Estatística (IBGE), no acumulado do ano foram abatidas 4,4 bilhões de unidades de frango no mercado brasileiro. Comparativamente ao ano anterior, houve aumento de $10,9 \%$ no volume abatido. O Paraná continuou liderando a produção e os abates de frango no país, sendo responsável por $25,32 \%$ da produção nacional. Houve crescimento do abate da ordem de $11,02 \%$, passando de 994,01 milhões de cabeças, em 2006, para 1,1 bilhão em 2007. No mesmo ano, depois do Paraná, o segundo maior Estado produtor de aves é Santa Catarina, responsável por $18,9 \%$ da produção, com 824,3 milhões de cabeças. O Rio Grande do Sul foi responsável pela produção e abate de 734 milhões de cabeças (IBGE, 2008).

Além da ocupação gerada com a criação de frangos de corte propriamente dita, ocorrem, no Paraná, outros 26.200 empregos no setor industrial (abate e processamento), serviços de logística de transporte de insumos, frangos e produtos finais. O complexo agroindustrial da avicultura de corte paranaense (áreas industrial, agrícola, de logística e comércio), gera mais de 50.000 empregos diretos e indiretos (SEAB/DERAL, 2005).

Atuam no setor, no Estado, 31 empresas (27 privadas e quatro cooperativas) que congregam, aproximadamente, oito mil avicultores (produtores rurais integrados). Das 28 empresas credenciadas junto ao Serviço de Inspeção Federal do Ministério da Agricultura e Pecuária, 16 estão habilitadas para a exportação de carnes e produtos avícolas. Os incubatórios e estabelecimentos de produção de matrizes totalizam 24 empresas, das quais 17 vinculadas às integrações avícolas e sete classificadas como independentes. Há, portanto, predominância absoluta do sistema de produção integrado (SEAB/ DERAL, 2005).

De acordo com Figueiredo et al. (2006), o sistema de produção integrado é o que tem se tornado mais presente no setor avícola brasileiro. Isso porque, a produção via contrato de integração, propicia benefícios tanto ao integrador quanto ao integrado. Para o primeiro, as vantagens se relacionam à redução de imobilização de capital em instalações e equipamentos, além de evitar encargos trabalhistas. Já para o integrado, as vantagens estão associadas ao recebimento de assistência técnica especializada, insumos e crédito, além de ter, ao final da criação, destino para a produção e, com isso, certeza de renda.

Contudo, embora o sistema de integração seja apontado como um mecanismo que reduz risco para o produtor, é necessário não esquecer que o frango é uma commodity e, portanto, está sujeito às condições e riscos de mercado, tanto do mercado de carnes quanto de milho e soja que são a base da ração desses animais. Outro fator que interfere na rentabilidade dos avicultores é a eficiência no manejo de cada lote.

Toda essa configuração do setor vai ao encontro de um paradigma que requer, cada vez mais, novos processos tecnológicos e produtivos, exigindo recursos econômicos, humanos e de planejamento.

Assim, a hipótese que embasa este estudo é que, como em toda atividade agropecuária, a avicultura incorre em riscos, o que pode comprometer seu desempenho, mesmo em mecanismo contratual que é o sistema integrado na avicultura.

Neste sentido, dada a importância do setor para a economia paranaense, bem como sua inserção em um contexto de riscos, objetiva-se neste trabalho proceder à análise econômica da produção de frango de corte, verificando as fontes de risco e sua influência na rentabilidade da atividade em três sistemas de produção: climatizado, automático e manual, na produção via integração no estado do Paraná.

\section{MATERIAL E MÉTODOS}

Em aplicações econômicas, a distinção entre risco e incerteza tem sido feita com base na disponibilidade de informações, que permitam mensurar a probabilidade de ocorrência de eventos futuros. Diz-se que há risco em uma atividade quando são conhecidos os possíveis valores assumidos por certa variável relevante e suas respectivas probabilidades de ocorrência. Quando as probabilidades de ocorrência ou estados futuros da variável não são conhecidos, diz-se que há incerteza (FIGUEIREDO et al., 2006).

De acordo com Heifner \& Coble (1999), citados por Esperancini (2006), as maiores fontes de risco sistemático na agricultura, ou seja, aquelas que podem de alguma forma ser objeto de previsão e quantificação são: produção, preços dos produtos e custos de produção. 
Outras fontes de risco podem guardar relação entre si (como, por exemplo, risco cambial e de preços, risco institucional influenciando riscos financeiros).

Sendo assim, neste trabalho é feita análise da rentabilidade da avicultura de corte associada às seguintes variáveis de risco: preço do produto, produtividade e custos de produção. As duas primeiras são componentes da receita e a última refere-se ao custo total de produção (custos fixos e variáveis do integrado e da agroindústria/ integradora).

Foram considerados três sistemas de produção (tipos de aviário): climatizado, automático e manual, na produção via integração no estado do Paraná. Segundo Canever et al. (1998), os galpões manuais empregam comedouros tubulares, bebedouros pendulares, cortinas de ráfia e a calefação é feita com campânulas a gás de operação manual. Os automatizados caracterizam-se por possuir comedouros tuboflex, bebedouros nipple, cortinas de ráfia e campânulas a gás sensorizadas. Já os climatizados são compostos por equipamentos computadorizados que regulam temperatura, umidade e velocidade do vento. Possuem, ainda, cortinas impermeáveis e as operações de alimentação, fornecimento de água e calefação automatizadas.

Para determinar a rentabilidade (renda líquida) e risco da produção de frango de corte no estado do Paraná, foi utilizado o método de simulação de Monte Carlo, através do programa @ Risk 4.5 (PALISADE CORPORATION, 2002).

O método de Monte Carlo é reconhecido como uma técnica válida e apresenta uma série de vantagens como redução de tempo, de custos e possibilidades de repetição, sob diferentes condições de produção. Ademais, ao contrário da análise determinística, que utiliza valores únicos para obtenção de um indicador do sistema, a técnica de simulação de Monte Carlo permite incorporar as possibilidades de alteração das variáveis, segundo as probabilidades de sua ocorrência (CRUZ, 1986).

As etapas realizadas nesse método são: 1) seleção e identificação das distribuições de probabilidades das variáveis em estudo; 2) seleção aleatória de um valor de cada variável em estudo, associada à probabilidade de sua ocorrência; 3) determinação do valor do indicador de desempenho do sistema utilizando o valor da variável associada à probabilidade de ocorrência; 4) repetição das etapas 2 e 3 até que a distribuição de probabilidade do indicador de rentabilidade satisfaça as exigências dos tomadores de decisão (AVEN, 2003).

Associou-se à rentabilidade três variáveis básicas: preços, produtividade e custos de produção.
Para os preços, a determinação de sua distribuição foi dada a partir uma série de preços médios nominais mensais de frango vivo recebidos pelos produtores ( $\mathrm{R} \$$ / $\mathrm{kg}$ ), no período de janeiro de 2000 a outubro de 2007 . Essa série de preços foi obtida junto ao Departamento de Economia Rural da Secretaria da Agricultura e do Abastecimento do Paraná (SEAB/DERAL), que procede à coleta junto aos Núcleos Regionais, sua estimativa e divulgação (SEAB/DERAL, 2007c). Os valores foram corrigidos pelo IGP-DI, com base em outubro de 2007. O preço do frango vivo é o mesmo, independente do tipo de aviário. Após o deflacionamento, foi determinada a distribuição de freqüência, com os melhores resultados estatísticos pelo critério qui-quadrado.

Para a produtividade, foram tomados três valores, considerando-se as situações de viabilidade (taxa de sobrevivência): $96,5 \%, 100 \%$ e de $95 \%$. A partir daí, com base em dados da Embrapa (2007), estimou-se a produtividade por lote (em $\mathrm{kg}$ ): número de aves alojadas $\mathrm{x}$ peso médio $\mathrm{x}$ viabilidade. Cada tipo de aviário aloja diferente número de aves: climatizado: 21.600 aves/lote; automático: 17.000 aves/lote; manual: 14.400 aves/lote. O peso médio do frango é de $2,3 \mathrm{~kg}$. Para os valores da produtividade optou-se por utilizar a distribuição triangular.

Para os custos, foi utilizada a estrutura de custo de produção de frango de corte disponibilizada e divulgada pela SEAB/DERAL e pela EMBRAPA Suínos e Aves (EMBRAPA, 2007; SEAB/DERAL, 2007b). Essa apresenta os custos fixos e variáveis mensais de produção do Produtor Integrado e da Agroindústria, por tipo de aviário, não incluindo os custos de instalação/implantação do aviário.

Inicialmente, procurou-se identificar os itens que tinham maior peso no custo total de produção (por lote). Tomando-se como base de referência a estrutura de custos de outubro de 2007, foi possível identificar os itens de custos que podem potencialmente gerar risco nos custos de produção. Verificou-se que são os custos variáveis que têm maior peso no custo total (CT) de produção $(94,50 \%$ do CT no climatizado; $95,30 \%$ no automático; $95,12 \%$ no manual). Observou-se, ainda, que dentro dos custos variáveis, dois itens se destacam: custos com "pintos" (21,61\% do CT no climatizado; $21,34 \%$ do CT no automático; 20,85\% do CT no manual) e custos com "ração" (56,97\% do CT no climatizado; $56,25 \%$ do CT no automático; 54,95\% do CT no manual). Diante disso, optouse por avaliar os riscos dos custos de produção desmembrados em: Custos Fixos, Custos Variáveis com Pintos, Custos Variáveis com Ração e Outros Custos Variáveis. Para todos os itens de custo foi tomada uma 
série de custos mensais ( $\mathrm{R} \$$ por lote), no período de janeiro de 2006 a outubro de 2007 (série disponibilizada pela fonte). Os valores foram corrigidos pelo IGP-DI, com base em outubro de 2007. Também nesse caso, foi determinada a distribuição de freqüência, identificada pelos melhores resultados estatísticos do critério qui-quadrado.

$\mathrm{O}$ indicador de rentabilidade foi dado pela expressão:

$$
R L=[P v Q-(C F+C V p+C V r+C V o)]
$$

Onde:

$\mathrm{RL}=\mathrm{f}(\mathrm{RL}) \rightarrow$ distribuição de probabilidade da receita líquida $(\mathrm{R} \$ /$ lote $)$

$\mathrm{Pv}=\mathrm{f}(\mathrm{P}) \rightarrow$ distribuição de probabilidade do preço do produto $(\mathrm{R} \$ / \mathrm{kg})$,

$\mathrm{Q}=\mathrm{f}(\mathrm{Qa}) \rightarrow$ distribuição de probabilidade $\mathrm{da}$ produtividade por lote ( $\mathrm{kg} / \mathrm{lote})$,

$\mathrm{CF}=\mathrm{f}(\mathrm{CF}) \rightarrow$ distribuição de probabilidade de custo fixo (R\$/lote)

$\mathrm{CVp} ; \mathrm{CVr} ; \mathrm{CVo}=\mathrm{f}(\mathrm{CV}) \rightarrow$ distribuição de probabilidade dos itens de custo variável (R/lote).

\section{RESULTADOS E DISCUSSÃO}

Diversos são os fatores que podem interferir no nível e variação da rentabilidade de um sistema de produção. Contudo, existem aqueles que influenciam de forma direta e que, portanto, precisam ser previstos, quantificados e acompanhados. Nesse sentido, foram feitas análises de sensibilidade e identificadas variáveis críticas, consideradas fontes de risco nos três sistemas de produção integrada de frango de corte: climatizado, automático e manual. Dentre essas variáveis foi possível fazer análise de risco sobre o preço do frango, produtividade e custos de produção.

A Tabela 1 apresenta um resumo dos resultados encontrados de variações da renda líquida, nos três sistemas de produção.

Os indicadores de risco mostram que o sistema climatizado eleva o risco de produção, como indicam a elevação das medidas de desvio padrão e variância, em comparação ao automático e manual. Por sua vez, percebese que o sistema automático apresenta risco mais elevado que o manual, considerando os valores de desvio padrão e variância.

Verifica-se que o sistema climatizado piora o resultado de renda líquida no cenário mais pessimista de preço, produtividade e custos, apresentando resultado negativo significativo em comparação aos sistemas automático e manual. Entretanto, no cenário mais otimista, o climatizado apresenta o melhor resultado de renda líquida. Também a renda líquida média apresenta-se melhor no climatizado.

Ao se comparar os sistemas automático e manual, verifica-se que o automático apresenta resultados melhores de renda líquida, tanto no cenário mais otimista (com valor máximo superior ao do manual), quanto no cenário mais pessimista (com renda líquida negativa menor).

Cabe destacar que a probabilidade de sucesso do climatizado é maior que dos outros dois sistemas, com

Tabela 1 - Resultados estatísticos da renda líquida da produção de frango de corte integrada no estado do Paraná, para os sistemas climatizado, automático e manual.

\begin{tabular}{lrrr}
\hline \multicolumn{1}{c}{ Indicadores } & Climatizado & Automático & \multicolumn{1}{c}{ Manual } \\
\hline Máximo & $30.651,26$ & $23.667,47$ & $20.206,49$ \\
Mínimo & $-22.627,11$ & $-12.247,94$ & $-14.233,58$ \\
Média & $8.462,40$ & $6.102,35$ & $3.934,78$ \\
Mediana & $8.786,26$ & $6.226,57$ & $4.085,15$ \\
Moda & $11.511,09$ & $6.153,44$ & $4.932,61$ \\
Chance de resultado positivo & $82,0 \%$ & $81,0 \%$ & $74,1 \%$ \\
Chance de resultado negativo & $18,0 \%$ & $19,0 \%$ & $25,9 \%$ \\
Desvio padrão & 8562,78 & 6721,63 & 5637,71 \\
Variância & 73321189,04 & 45180339,41 & 31783778,16 \\
Iterações & 5000 & 5000 & 5000 \\
Erros & 0 & 0 & 0 \\
\hline
\end{tabular}

Fonte: Resultados da pesquisa. 
chances de resultados positivos superiores. O sistema manual é o que apresenta maior probabilidade de obter-se renda líquida negativa.

Na Tabela 2, os resultados da análise de sensibilidade mostra que nos três sistemas a rentabilidade tem grande sensibilidade ao preço do frango, apresentando também alta correlação positiva com essa variável. Verifica-se, então, que variações na renda líquida na produção integrada de frango de corte dá-se, principalmente, por oscilações no preço do produto. A variável preço do produto mostra-se, desse modo, como a principal fonte de risco.

A produtividade (item componente da receita) apresentou-se menos relevante que o preço, na determinação de variações da renda líquida. Como o preço, a produtividade tem, como esperado, correlação positiva com a renda líquida.

Depois do preço do produto, os custos variáveis com ração e com pintos são as variáveis que mais afetam a renda líquida. Esses, naturalmente, apresentam correlação negativa com a rentabilidade. Os outros itens de custo (custo fixo e outros custos variáveis) mostraram-se menos importantes que os custos variáveis com ração e pintos e, como esses últimos, mantêm correlação negativa com a renda líquida (Tabela 2 ).

O mapeamento de risco dado pelos percentis mostra que, quanto menor o nível de risco, há possibilidade de menores níveis de renda líquida e quanto maior o risco, maior a renda.Verifica-se que os sistemas climatizado e automático apresentam risco de prejuízo na produção em níveis porcentuais de risco menor que o sistema manual (Tabela 3 ).

Até o nível de $5 \%$ de risco, o prejuízo (renda líquida negativa) é superior no sistema climatizado. Até $10 \%$ de risco esse sistema ainda apresenta prejuízo ligeiramente superior ao do automático. Contudo, acima desse nível de risco, o prejuízo nesse sistema mostra-se menor em comparação aos demais sistemas, e começa a apresentar resultado positivo em nível de risco menor que os sistemas automático e manual. A esse respeito, observa-se que a $20 \%$ de risco, tanto o climatizado quanto o automático apresentam renda líquida positiva, observando-se, contudo, que a presença de resultado positivo acontece antes para o climatizado (acima de $18 \%$ de risco). Para menores níveis de risco (até 5\%), o sistema climatizado mostra-se pior que os outros dois. Entretanto, à medida que o grau de risco aumenta, esse sistema apresenta melhores resultados.

O sistema manual é o que apresenta prejuízo até o nível de risco superior a $25 \%$. Somente a partir daí é que a renda líquida passa a ser positiva.

O maior retorno possível (ao nível de $100 \%$ de risco) ocorre no sistema climatizado, podendo chegar a $\mathrm{R} \$ 30.651,26 /$ lote, enquanto no automático pode atingir $\mathrm{R} \$$ 23.667,47/lote e, no manual, R\$20.206,49/lote.

Para melhor ilustrar esses resultados, nas Figuras 1, 2 e 3 são apresentadas as distribuições de probabilidade acumulada de renda líquida dos sistemas climatizado, automático e manual.

Pela Figura 1, é possível observar que existe a probabilidade de $5 \%$ de que a renda líquida seja menor ou igual a-R \$6.200,73 (prejuízo), e 95\% que seja igual ou menor a $\mathrm{R} \$ 21.958,88$. Verifica-se, portanto, que o sistema climatizado apresenta probabilidade de $5 \%$ de que a renda líquida seja superior a $\mathrm{R} \$ 21.958,88$.

A Figura 2 apresenta a distribuição de probabilidade da renda líquida do sistema automático.

Pela Figura 2, é possível observar que existe a probabilidade de $5 \%$ de que a renda líquida seja menor ou igual a -R \$5.273,47 (prejuízo), e 95\% que seja igual ou menor a $\mathrm{R} \$ 16.708,92$. Verifica-se, portanto, que existe a probabilidade de $90 \%$ que a renda líquida se situe nesse intervalo.

A Figura 3 apresenta a distribuição de probabilidade da renda líquida do sistema manual.

Tabela 2 - Análise de sensibilidade da renda líquida da produção de frango de corte integrada no estado do Paraná, para os sistemas climatizado, automático e manual.

\begin{tabular}{lcccccc}
\hline \multirow{2}{*}{ Variáveis } & \multicolumn{2}{c}{ Climatizado } & \multicolumn{2}{c}{ Automático } & \multicolumn{2}{c}{ Manual } \\
\cline { 2 - 7 } & Regressão & Correlação & Regressão & Correlação & Regressão & Correlação \\
\hline Preço & 0,894 & 0,892 & 0,885 & 0,898 & 0,882 & 0,895 \\
Produtividade & 0,094 & 0,090 & 0,094 & 0,093 & 0,111 & 0,130 \\
Custo Fixo & $-0,107$ & $-0,034$ & $-0,033$ & $-0,024$ & $-0,017$ & $-0,027$ \\
CV Ração & $-0,307$ & $-0,301$ & $-0,305$ & $-0,289$ & $-0,309$ & $-0,306$ \\
CV Pintos & $-0,299$ & $-0,272$ & $-0,304$ & $-0,301$ & $-0,300$ & $-0,282$ \\
Outros CV & $-0,069$ & $-0,031$ & $-0,079$ & $-0,061$ & $-0,089$ & $-0,064$ \\
\hline$R$-Squared & 0,999924 & \multicolumn{5}{c}{0,999907} \\
\hline
\end{tabular}

Fonte: Resultados da pesquisa. 
MELO, C. O. de et al.

Tabela 3 - Percentis de risco da renda líquida da produção de frango de corte integrada no estado do Paraná, para os sistemas climatizado, automático e manual.

\begin{tabular}{crrr}
\hline Percentis de risco $(\%)$ & Climatizado $(\mathrm{R} \$ /$ lote $)$ & Automático $(\mathrm{R} \$ /$ lote $)$ & Manual $(\mathrm{R} \$ /$ lote $)$ \\
\hline 0 & $-22.627,11$ & $-12.247,94$ & $-14.233,58$ \\
5 & $-6.200,73$ & $-5.273,47$ & $-5.635,65$ \\
10 & $-3.233,00$ & $-3.005,52$ & $-3.683,87$ \\
15 & $-1.108,42$ & $-1.305,35$ & $-2.320,85$ \\
20 & 885,85 & 36,69 & $-1.165,27$ \\
25 & $2.397,08$ & $1.321,05$ & $-79,28$ \\
30 & $3.715,70$ & $2.412,43$ & 798,62 \\
35 & $5.095,89$ & $3.371,77$ & $1.778,97$ \\
40 & $6.311,74$ & $4.357,59$ & $2.598,20$ \\
45 & $7.635,01$ & $5.303,26$ & $3.354,00$ \\
50 & $8.786,26$ & $6.226,57$ & $4.085,15$ \\
55 & $10.032,93$ & $7.200,81$ & $4.860,95$ \\
60 & $11.170,07$ & $8.113,30$ & $5.719,95$ \\
65 & $12.377,25$ & $9.119,23$ & $6.464,90$ \\
70 & $13.582,10$ & $10.180,79$ & $7.224,05$ \\
75 & $14.967,78$ & $11.185,44$ & $8.075,41$ \\
80 & $16.398,31$ & $12.197,59$ & $9.067,07$ \\
85 & $17.816,06$ & $13.376,94$ & $10.020,29$ \\
90 & $19.553,91$ & $14.782,73$ & $11.216,86$ \\
95 & $21.958,88$ & $16.708,92$ & $12.867,44$ \\
100 & $30.651,26$ & $23.667,47$ & $20.206,49$ \\
\hline
\end{tabular}

Fonte: Resultados da pesquisa.

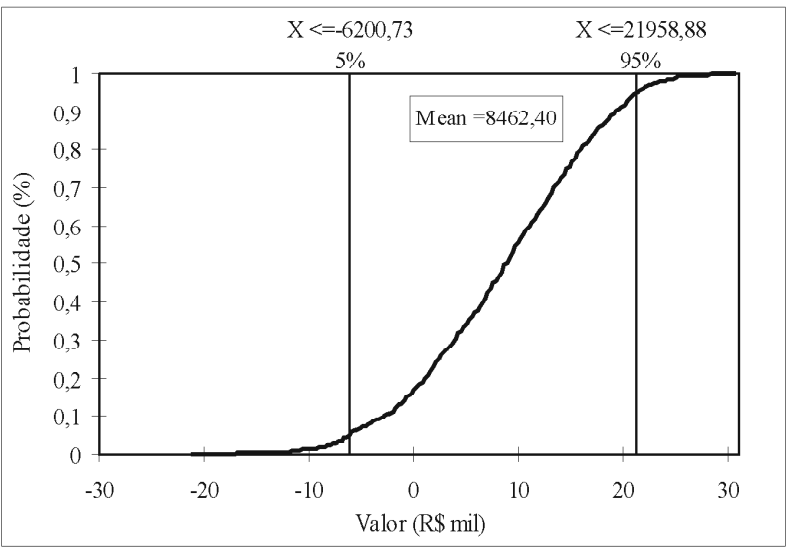

Fonte: Resultados da pesquisa.

Figura 1 - Distribuição de probabilidade acumulada de valores da renda líquida da produção de frango de corte integrada no estado do Paraná, para o sistema climatizado.

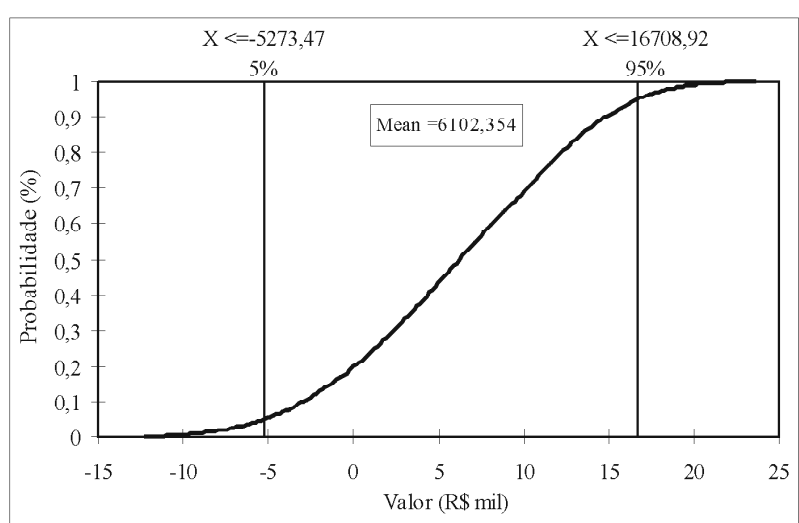

Fonte: Resultados da pesquisa.

Figura 2 - Distribuição de probabilidade acumulada de valores da renda líquida da produção de frango de corte integrada no estado do Paraná, para o sistema Automático. 


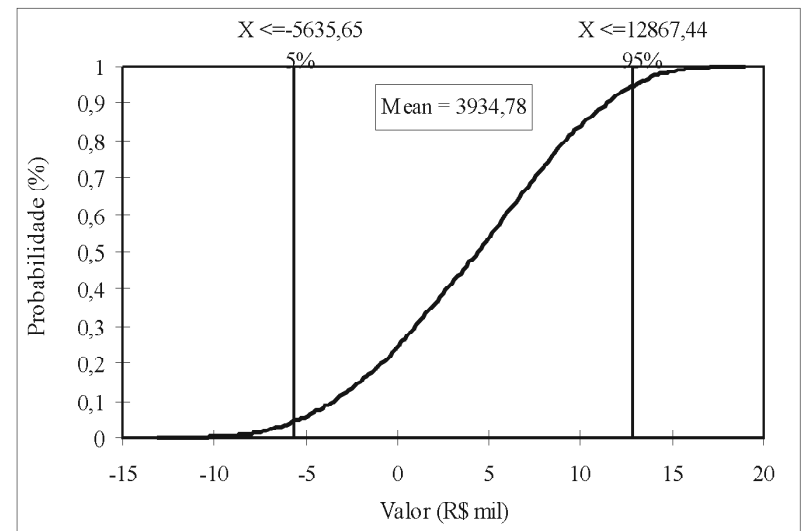

Fonte: Resultados da pesquisa.

Figura 3 - Distribuição de probabilidade acumulada de valores da renda líquida da produção de frango de corte integrada no estado do Paraná, para o sistema manual.

Pela Figura 3, é possível observar que existe a probabilidade de $5 \%$ de que a renda líquida seja menor ou igual a-R $\$ 5.635,65$ (prejuízo), e 95\% que seja igual ou menor a $\mathrm{R} \$ 12.867,44$. Verifica-se, portanto, que existe a probabilidade de $90 \%$ que a renda líquida se situe nesse intervalo. A possibilidade que a renda líquida no sistema manual seja superior a $\mathrm{R} \$ 12.867,44$ é da ordem de 5\%.

Esses resultados sugerem que, apesar de o contrato de integração mostrar-se interessante tanto para o integrado quanto para o integrador, ele não elimina por completo os riscos, especialmente os associados ao preço do produto. Observando os sistemas de produção (tipos de aviário), é possível inferir que, para níveis de risco de até 5\%, o climatizado mostra-se mais propenso a prejuízos maiores. Já à medida que os níveis de risco aumentam, os resultados são mais interessantes nesse sistema. Ademais, na probabilidade de $90 \%$, o intervalo de possíveis valores de renda líquida é mais interessante no climatizado.

A produção de frango de corte no estado do Paraná constitui-se numa atividade importante econômica e socialmente. A produção integrada é, praticamente, a adotada em todo o Estado, podendo ser interessante para o produtor rural integrado e para a agroindústria integradora. Contudo, a inexistência de outra forma de organização da produção e a dimensão que tal estrutura alcançou pode impor ao produtor rural a aceitação desse tipo de arranjo produtivo, sem possibilidades de optar por outro modelo. Cabe ainda observar que os valores contratuais que o integrado recebe da integradora estão relacionados à eficiência da produção e, portanto, ao manejo. A adoção de técnicas e tipos de aviários mais modernos podem contribuir para melhorar o desempenho produtivo.

Mesmo assim, com os resultados constatou-se que a atividade é passível de riscos. Ao analisar-se o processo produtivo como um todo, avaliando variáveis referentes a preços do produto (e não somente à parte recebida pelo integrado), custos totais de produção (do integrado e da integradora), foi possível identificar as principais fontes de risco, bem como a sensibilidade da renda líquida.

A principal fonte de riscos é o preço do produto, seguida dos custos variáveis (especialmente com ração e pintos). Como o preço pode muitas vezes ser uma variável fora do controle do produtor, interessante se faz atentar para formas que permitam um monitoramento dos custos de produção, no sentido de serem os menores possíveis, através da eficiência no manejo, adoção de tecnologias, pesquisas para maior rendimento/ conversão alimentar.

\section{CONCLUSÕES}

Em relação aos sistemas de produção analisados, pode-se perceber que o climatizado, a níveis mais baixos de risco, apresenta prejuízo maior. Contudo, à medida que o risco aumenta, os resultados são melhores que dos sistemas automático e manual, configurando como uma opção interessante para o produtor com menor aversão ao risco.

Cabe mencionar que as mudanças ocorridas na economia nacional e internacional forçam a modernização dos processos produtivos e, inserida nesse contexto, a cadeia avícola de corte não é exceção. Num ambiente de maior estabilidade monetária brasileira e de abertura econômica, nova configuração das unidades produtivas passa a ser uma exigência. A introdução de novas tecnologias e formas de produção do setor avícola vai ao encontro desse paradigma, de modo que a adoção de sistemas mais tecnificados parece ser uma tendência na avicultura de corte.

\section{REFERÊNCIAS BIBLIOGRÁFICAS}

AVEN, T. Foundations of risk analysis. West Sussex: J. Wiley, 2003. 190 p.

BARROS, A. R. Avicultura: relatório setorial integrante do projeto "Economia de Pernambuco: uma contribuição para o futuro". Recife: Secretaria de Planejamento, 2005.

CANEVER, M. D.; SANTOS FILHO, J. I.; CHICUCHETTA, O.; TALAMINI, D. J. D. Mudanças tecnológicas na avicultura do oeste catarinense. In: CONFERÊNCIAAPINCO DE CIÊNCIA E TECNOLOGIAS AVÍCOLAS, 1998, Concórdia. Anais... Concórdia: Embrapa Suínos e Aves, 1998. 
CRUZ, E. R. Aspectos teóricos sobre a incorporação de riscos em modelos de decisão. In: CONTINI, E. et al. Planejamento da propriedade agrícola: modelos de decisão. 2. ed. Brasília, DF: Embrapa, 1986.

EMPRESA BRASILEIRA DE PESQUISA AGROPECUÁRIA. Custos de produção de frango de corte: série de dados. Disponível em: <http:// 'WWW.cnpsa.embrapa.brì. Acesso em: 22 nov. 2007.

ESPERANCINI, M. S. T. Avaliação econômica de sistemas de sucessão de culturas sob condições de risco no estado de São Paulo, 2005. 2006. Tese (Livre Docência) Universidade Estadual Paulista "Júlio de Mesquita Filho", Botucatu, 2006.

FIGUEIREDO, A. M.; SANTOS, P. A.; SANTOLIN, R.; REIS, B. S. Integração na criação de frangos de corte na microrregião de Viçosa-MG: viabilidade econômica e análise de risco. Revista de Economia e Sociologia Rural, Brasília, v. 44, n. 4, out./dez. 2006.

INSTITUTO BRASILEIRO DE GEOGRAFIA E ESTATÍSTICA. Indicadores IBGE: estatística de produção pecuária. Brasília, DF, 2008.
PALISADE CORPORATION. @RISK 4.5 for industrial edition. New York, 2002.

SECRETARIA DA AGRICULTURA E DO ABASTECIMENTO DO PARANÁ/DEPARTAMENTO DE ECONOMIA RURAL. Paraná: perfil da produção de frangos de corte. Curitiba, 2005. Disponível em: <http:// Www.seab.pr.gov.birl

SECRETARIADAAGRICULTURAE DOABASTECIMENTO DO PARANÁ/DEPARTAMENTO DE ECONOMIA RURAL. Valor bruto da produção agropecuária paranaense em 2005. Curitiba, 2007a. Disponível em: <http://Www.seab.pr.gov.br̀'. Acesso em: 21 nov. 2007.

SECRETARIA DA AGRICULTURA E DO ABASTECIMENTO DO PARANÁ/DEPARTAMENTO DE ECONOMIA RURAL. Estimativas de custo de produção: frango de corte. Curitiba, 2007b. Disponível em: <http:// 'Www.seab.pr.gov.bril'. Acesso em: 22 nov. 2007.

SECRETARIA DA AGRICULTURA E DO ABASTECIMENTO DO PARANÁ/DEPARTAMENTO DE ECONOMIA RURAL/DIVISÃO DE ESTATÍSTICAS BÁSICAS. Preços médios nominais mensais recebidos pelos produtores no Paraná. Curitiba, 2007c. Disponível em: <http://Www.seab.pr.gov.brr. Acesso em: 22 nov. 2007. 